Proc. Indian Acad. Sci. (Chem. Sci.), Vol. 108, No. 6, December 1996, pp. 593-604.

(C) Printed in India

\title{
Molecular-level functionalization of electrode surfaces. An overview
}

\author{
V YEGNARAMAN \\ Electrodics and Electrocatalysis Division, Central Electrochemical Research Institute, \\ Karaikudi 630 006, India
}

\begin{abstract}
Electrochemical reactions occur at electrode/electrolyte interfaces. Hence, manipulation and design of electrochemical interfaces accompanied by surface modifications have assumed vital importance. Molecular level modification, either at the monolayer or multilayer level of electrode surfaces and leading to functionalization of electrodes, is being actively pursued by researchers. Modification based on the self-assembled monolayer approach has enabled electrodes to acquire molecular recognition and molecular electronic characteristics. Functionalization of electrode surfaces using polymeric materials and enzymes has facilitated electrodes in exhibiting properties like catalysis, molecular recognition, electrochromism and birefringence. The results of such molecular level functionalization studies of electrode surfaces carried out recently in our laboratories are presented in this overview. Besides, some representative results reported from elsewhere are also included.
\end{abstract}

Keywords. Electrode/electrolyte interface; surface modification; molecular-level functionalization of electrodes.

\section{Introduction}

Many interesting and important phenomena occur at interfaces. The electrode/electrolyte interface is of use in a variety of industrially important processes like energy conversion, corrosion of metals, electroplating, electrocatalysis and electroanalysis. The electrochemical interface denotes a dimensionally small region comprising (i) the top layer of atoms on the electrode, (ii) the layer of adsorbed solvent, specifically adsorbed ions, molecules and monolayer films, and (iii) the region consisting of the diffuse layer and the modifying overlayers, if any. The dimensions of these are: (i) of the order of a few angstroms in metals and microns in semiconductors, (ii) of the order of a few angstroms, and (iii) can vary from nanometers to microns. Essentially all electrochemical reactions occur at these interfaces and hence the interfacial region plays a vital role. Manipulation and design of electrochemical interfaces involving electrodes with surface modification and investigations of electrochemical interfaces using such modified electrodes pursued intensively for the last two to three decades have emerged as a fascinating and fruitful area of research with great potential for applications in all areas of electrochemistry.

Recent developments in the design of electrochemical interfaces especially through mono or muitilayers of organic or inorganic species have offered means for precise control of electrode reactivity. Rational design of the interface, aimed at better understanding and control of the electrochemical reaction kinetics, and attainment of improved sensitivity and selectivity to reactions can be realized by manipulating the electrode surface or the interfacial region. The former approach involving the use of 
metal/semiconductor and polycrystalline/single crystal materials as electrodes will not be included in this discussion. The latter involves attachment of molecular species (either as a monolayer or as a multilayer) to the electrode surface with the objective of transferring the known chemical or electrochemical reactivity of the attached molecular species to the electrochemical interface. This approach, involving functionalization of electrode surfaces, is the focus of several researchers currently because of the ease with which it facilitates achievement of structurally known and coherently organized molecular films on electrode surfaces, through Langmuir-Blodgett or self-assembly methods and the use of organic/inorganic polymers as host matrices. Some recent developments in these areas reported from our laboratory and elsewhere will be discussed. For an exhaustive review, readers are advised to see the biennial reviews of Analytical Chemistry.

Further, in the functionalization of electrode surfaces, molecular level interactions can occur either in binding the modifying species to the electrode surface or in host-guest interactions between the reactant species in solution and the surface-immobilized modifier species. Examples of both types will be discussed here.

\section{Self-assembled monolayers}

Formation of a molecular monolayer through self-assembly results from the chemical binding between the molecule and the substrate surface, and the microstructural organization of the assembled monolayer. Examples include alkylsilanes attached to glass surfaces (Sagiv 1980) and thiols (Porter et al 1987; Whitesides and Labinis 1990; Walczak et al 1991; Finklea and Hanshew 1992) and disulphides (Troughton et al 1988) tethered to gold surfaces.

Self-assembled monolayers (SAM) of alkane thiols and substituted alkane thiols on gold surfaces have been advantageously exploited for bringing about molecular level functionalization (MLF), over the last decade. SAMs of alkane thiols of the type X$\left(\mathrm{CH}_{2}\right)_{n}$-SH where ' $\mathrm{X}$ ' may be an alkyl or a functional group, have yielded densely packed and defect-free monolayers ( $c f$ figure 1). Probing by different techniques has revealed that the binding of these thiols to the gold surface is due to strong covalent linkage between the $\mathrm{Au}$ atom and the $\mathrm{S}$ of the thiol molecule, leading to the formation of Au thiolate bonds (Walczak et al 1991). Through judicious choice of the ' $X$ ' group in the thiol molecule, desired functionality is imparted to the electrode. Typical investigations involving MLF of electrode surfaces through SAM are briefly discussed below.
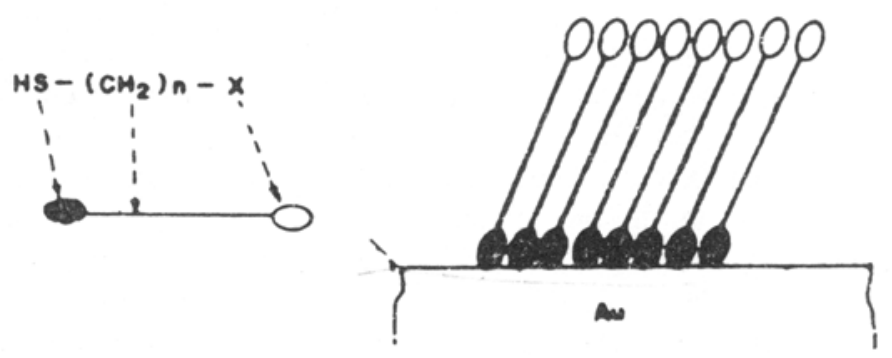

Figure 1. A schematic description of the formation of self-assembled monolayer of $\mathrm{X}-\left(\mathrm{CH}_{2}\right)_{n}-\mathrm{SH}$ on Au surface. 


\subsection{Electron transfer kinetics}

SAMs that are covalently and virtually irreversibly attached to the electrode surface have enabled further understanding of heterogeneous electron transfer. Many parameters of electron transfer, in the light of the Marcus theory, including distance dependence and reorganization energy, were examined using SAMs $\omega$-hydroxyalkane thiols and thiols with pendent redox groups on gold. The voltametric investigations (Finklea and Hanshew 1992) of SAMs of the thiols of the type $\mathrm{HS}-\left(\mathrm{CH}_{2}\right)_{n}-\mathrm{CONH}-\mathrm{CH}_{2}$ pyRu( $\left.\mathrm{NH}_{3}\right)_{5}^{2+/ 3+}$ $(n=10,11,15)$ have shown that the redox centres exhibit ideal surface reversible behaviour. The standard rate constant for electron transfer with the electroactive monolayer is found to be significantly influenced by the distance between the electrode surface and the redox centre (i.e.) the alkyl chain length determined by the number of methylene groups in the tether. As ' $n$ ' varies from 10 to 15 , the standard rate constant decreases by about four orders. Successful attempts have been made to obtain data on the densities of electronic states by measuring the redox kinetics on $\mathrm{Au}$ electrodes functionalized with $\omega$-hydroxy thiol monolayers (Miller and Gratzel 1991). Again, investigations of ferro/ferricyanide systems on Au electrodes functionalized with SAMs of $\omega$-hydroxy thiol have shown that electron transfer takes place through electron tunnelling (Miller et al 1991). Voltametric studies of the redox species of terminally substituted alkane thiol monolayer assembly have helped to understand the contribution of the diffuse layer potential and the electrostatic interactions between the terminal group and the redox species (Takehara and Takemura 1995).

\subsection{Molecular recognition}

This is another important area of MLF where monolayer modification has proved successful. Monolayer assembly consisting of $2,2^{\prime}$-thiobis (ethylacetoacetate) (TBEA) and $n$-octadecyl mercaptan on Au surface has exhibited ion selective characteristics. The TBEA sites, being more specific to coordination with $\mathrm{Cu}$ (II) led to its selective electroreduction in the presence of ferric ions, thereby exhibiting molecular recognition to cupric ions (Rubinstein et al 1988).

SAMs of alkane thiols with suitable functional tails, have enabled molecular discrimination based on electrostatic interactions between the functional group and the analyte species in solution. A monolayer of thioctic acid molecules self-assembled on gold exhibits molecular recognition to $\mathrm{Fe}(\mathrm{CN})_{6}^{3-/ 4-}$ and $\mathrm{Ru}\left(\mathrm{NH}_{3}\right)_{6}^{3+/ 2+}$ depending upon the $p \mathrm{H}$ of the electrolyte solution (Cheng and Toth 1992). At low $p \mathrm{H}$, the redox behaviour of the ferro/ferricyanide is reversibly observed, while that of ruthenium hexammine is significantly suppressed. At neutral and high $p H$, the redox behaviour of ferro/ferricyanide is totally suppressed while that of the other is reversible. This arises due to $\mathrm{pH}$-dependent dissociation or protonation of the carboxyl group of the thioctic acid, which exerts molecular recognition due to electrostatic interactions. Similarly, MLF of electrode surface through SAM has enabled selective estimation of the neurotransmitter, dopamine, in presence of a large excess of ascorbic acid (Malem and Mandler 1993). Molecular recognition of alkali metal cations by a nickel hexacyanoferrate monolayer anchored to SAMs of 3,3'-thiodipropionic acid on gold has been achieved (Bharathi et al 1995). The gold surface has been modified with a monolayer of 3,3'thiodipropionic acid by self-assembly. The $p H$ sensitive TPA monolayer is further derivatized to form a monolayer of nickel hexacyanoferrate as evidenced by the cyclic voltammograms described in figure 2 . The functionalized electrode shows molecular 


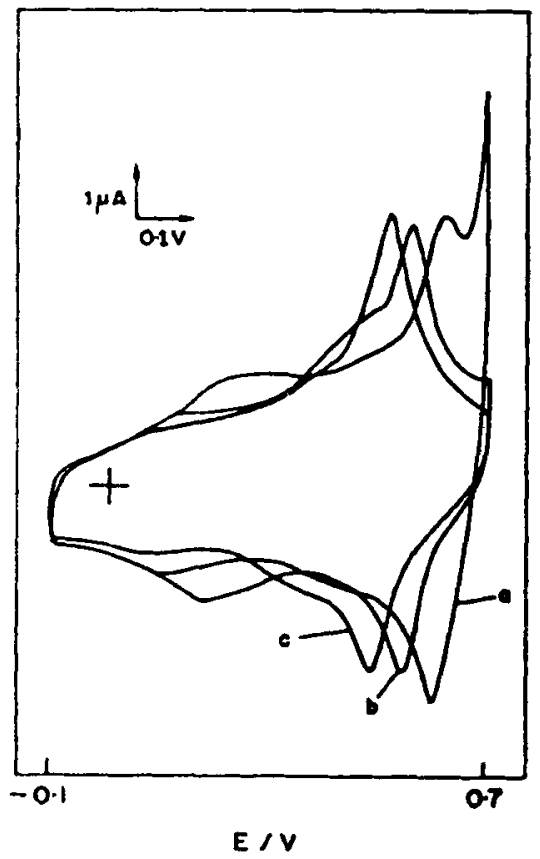

Figure 2. Cyclic voltammetric response of a monolayer of NiHCF anchored to Au through a self-assembled monolayer of 3,3'-thiodipropionic acid in $1 \mathrm{M}$ (curve a), $0.1 \mathrm{M}$ (curve b) and $0.01 \mathrm{M}$ (curve c) $\mathrm{KCl}$ solutions at $100 \mathrm{mV} / \mathrm{s}$. [Reprinted from: Bharathi et al, Langmuir (1995) 11 666-668, with the permission of the American Chemical Society.]

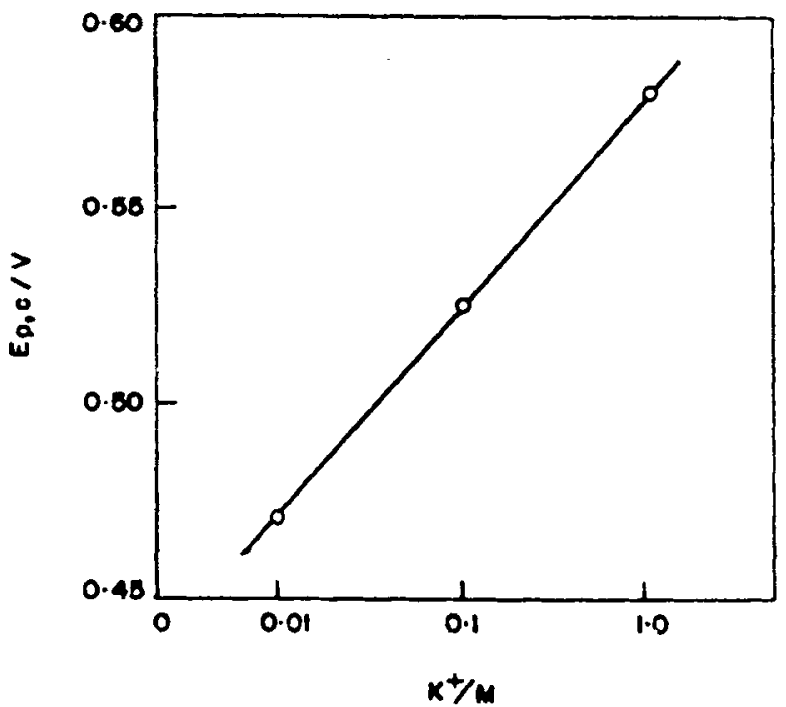

Figure 3. A plot of $E_{p, c}$ vs $\log \left[\mathrm{K}^{+}\right]$showing the near-Nernstian response of the $\mathrm{NiHCF}$ monolayer anchored to Au through a self-assembled monolayer of $3,3^{\prime}$ thiodipropionic acid to $\mathrm{K}^{+}$ions. [Reprinted from: Bharathi et al, Langmuir (1995) 11 666-668, with the permission of the American Chemical Society.] 


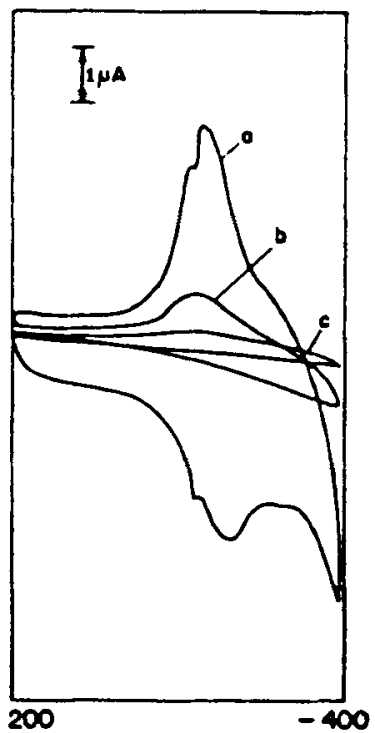

Figure 4. Cyclic voltammograms for the $\mathrm{Cu}$ UPD on "opened up" MBT monolayer-covered Au electrode in $1 \mathrm{M} \mathrm{H}_{2} \mathrm{SO}_{4}$ containing $1 \mathrm{mM} \mathrm{Cu}{ }^{2+}$ at $50 \mathrm{mV} / \mathrm{s}$. (a) First scan after "opening up", (b) intermediate scan and (c) after $20 \mathrm{~min}$ of cycling. [Reprinted from: Bharathi et al, Langmuir (1993) 9 1614-1617, with the permission of the American Chemical Society.]

discrimination between $\mathrm{K}^{+}$and $\mathrm{Na}^{+}$and exhibits a near-Nernstian response with a slope of $55 \mathrm{mV} /$ decade to $\mathrm{K}^{+}$in the range of $10^{-2}$ to $1 \mathrm{M}$ ( $c f$ figure 3 ).

\subsection{Molecular electronics}

This is a fast emerging field with potential applications leading to the design of molecular devices culminating in the development of a possible biocomputer. Of late, a number of reports describing active components for the fabrication of possible molecular devices are appearing. The fact that electrochemistry has a dominant role to play in this field is becoming increasingly evident (Hong 1989). MLF has helped to realize electrochemical interfaces with predesigned interfacial architecture that can impart properties like switching, gating, rectification etc.

A potential dependent 'opening' and 'closing' behaviour (Bharathi et al 1993) has been exhibited by the gold electrode by tethering it to a SAM of 2-mercaptobenzthiazole (MBT). The MBT covered Au electrode (Au/MBT) does not hinder the redox kinetics of ferro/ferricyanide; but it totally blocks the under-potential deposition (UPD) of copper. Interestingly, the $\mathrm{Au} / \mathrm{MBT}$ electrode when cycled between 0 and $1.3 \mathrm{~V}$ (vs. mercury/mercurous sulphate reference electrode [MSE]) 'opens up' and allows $\mathrm{Cu}$ UPD to take place virtually unhindered. Again, the opened up electrode, when cycled between -0.4 and $+0.4 \mathrm{~V}$ (vs MSE) 'closes in' and blocks the Cu UPD ( $c f$ figure 4 ). This 'opening' and 'closing' of the Au/MBT electrode could be repeated a number of times; however, the switching time is considerably high (about 15 minutes). This reversible potential-dependent gating behaviour has been tentatively explained in terms of a structural mechanism. It has been attributed to a possible potential dependent switching in 
<smiles>Sc1nc2ccccc2s1</smiles>

MBT

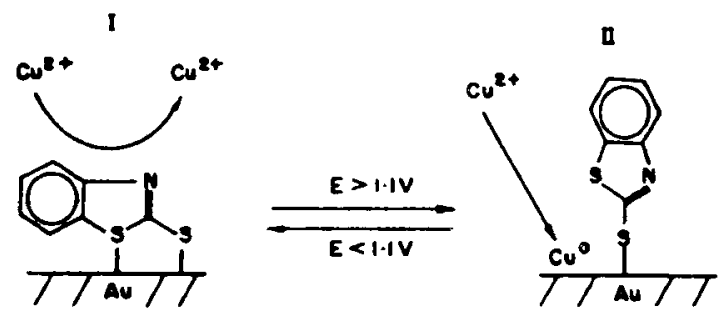

Figure 5. Scheme showing the possible structural mechanism for the "opening" and "closing" of MBT monolayer. (I) both S-atoms bound to the Au surface and (II) only one S-atom bound to the Au surface. [Reprinted from: Bharathi et al, Langmuir (1993) 9 1614-1617, with the permission of the American Chemical Society.]

the structural orientation (lateral/vertical depending upon both or one sulphur atom of MBT, being linked to Au surface) of the self-assembled MBT molecules ( $c f$ figure 5).

The self-assembly approach has added another new and interesting dimension to MLF by facilitating the formation of a monolayer of semiconductor nanocrystals on metal surfaces (Colvin et al 1992). Using the SAM of dithiols on gold and aluminium surfaces, a monolayer of cadmium sulphide nanocrystals bound to metal surfaces has been achieved. These cluster-on-metal systems hold promise for the design of molecular level photoelectrochemical devices.

\section{Polymer modified electrodes}

Functionalization of electrodes with polymeric modification is an area of active research since it has vast potential applications in fields such as electrocatalysis, sensors, electrochemical devices, ion exchange and photoelectrochemistry. Both inorganic and organic polymeric networks have been employed for this purpose. Conducting organic polymers are the popular examples of the latter category, while metal hexacyanoferrates (MHCF) and zeolitic clays belong to the former category.

\subsection{Metal hexacyanoferrate modified electrodes}

Electrode modification with MHCF has gained importance in view of the stability of the modified electrodes and their significant functional characteristics (Itaya et al 1986; Kulesza and Doblhofer 1989; Joseph et al 1992). Iron hexacyanoferrate, also called Prussian Blue (PB), and its analogues have been investigated by several researchers. The MHCF modification on different substrate electrodes has been achieved by both chemical and electrochemical methods. The modified electrodes, through specific molecular level interactions with the guest molecules, exhibit functional characteristics that have implications in processes like catalysis, electrochromism, molecular recognition, 
photoelectrochemical response etc. Salient features of some of these investigations are described below.

Thin films of PB on different substrates can be deposited chemically (Neff 1978) or electrodeposited by potentiostatic (Itaya et al 1982), galvanostatic (Kellawi and Rossiensky 1982) and potential cycling (Ellis et al 1981) methods. Such PB modified electrodes have been investigated for a variety of application areas like electrocatalysis (Itaya et al 1984), ion selectivity (Krishnan et al 1991), electrochromism (Mortimer and Rossiensky 1983; Kobayashi et al 1990) and photoresponse (Kaneko et al 1985). Useful functional characteristics of the PB arise possibly from its zeolitic structure (Itaya et al 1986) and its two reversible redox centres (Ellis et al 1981), which correspond to the oxidation of Prussian White (PW) to PB at about $0.2 \mathrm{~V}$ (vs SCE) and oxidation of PB to Berlin Green (BG) at about $0.8 \mathrm{~V}$ as per the following scheme.

At $0.2 \mathrm{~V}$

$$
\mathrm{K}_{4} \mathrm{Fe}_{4}\left[\mathrm{Fe}(\mathrm{CN})_{6}\right]_{3}-4 \mathrm{~K}^{+}-4 e^{-} \rightleftharpoons \mathrm{Fe}_{4}\left[\mathrm{Fe}(\mathrm{CN})_{6}\right]_{3} \text {. }
$$

At $0.8 \mathrm{~V}$

$$
\mathrm{Fe}_{4}\left[\mathrm{Fe}(\mathrm{CN})_{6}\right]_{3}-3 e^{-}+3 \mathrm{~A}^{-} \rightleftharpoons \mathrm{Fe}_{4}\left[\mathrm{Fe}(\mathrm{CN})_{6}\right]_{3} .
$$

The structures of PB and its analogues allow only alkali metal cations in their channelled structures. The entry and exit of cations during oxidation/reduction necessitated by the electroneutrality considerations of the film have been studied. Attempts to correlate selectivity for different cations vis-a-vis the diameter of their hydrated cations appear in the literature but are not fully substantiated. Nevertheless, the molecular recognition characteristics of PB and its analogues hold the promise of being technologically exploitable.

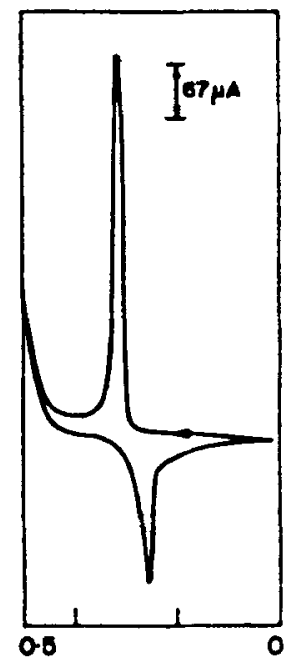

E/V VINCE

Figure 6. Cyclic voltammetric response of nickel hydroxide-derivatized Pt in $0.5 \mathrm{M}$ $\mathrm{KOH}$ at $5 \mathrm{mV} / \mathrm{s}$. [Reprinted from: Joseph et al, Solar Energy Mater. (1991) 23 1-5, with the permission of Elsevier Science-NL, The Netherlands.] 


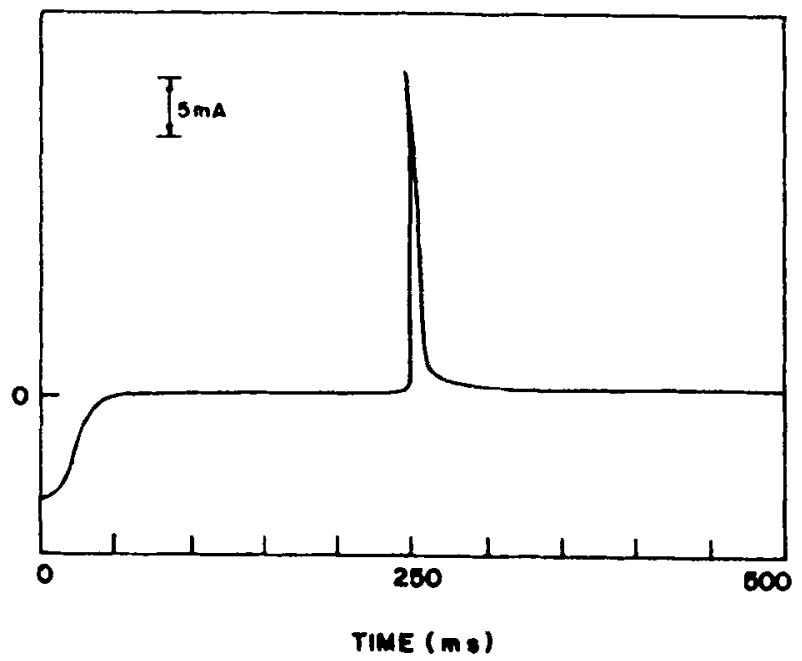

Figure 7. i-t Transient of nickel hydroxide-derivatized Pt $(0.5 \mathrm{~cm}$ long wire of $0.5 \mathrm{~mm}$ diameter) in $0.5 \mathrm{M} \mathrm{KOH}$, 'corresponding to the potential step of $0.0 \mathrm{~V}$ to $+0.5 \mathrm{~V}$ and back to $0.0 \mathrm{~V}$; pulse width: $250 \mathrm{~ms}$. [Reprinted from: Joseph et al, Solar Energy Mater. (1991) 23 1-5, with the permission of Elsevier Science-NL, The Netherlands.]

Another interesting functional characteristic of PB film is its ability to exhibit electrochromism. The PB film's ability to change its optical absorption characteristics with applied voltage in a stable and reversible manner (over $10^{5}$ cycles) has resulted in its use in electrochromic devices (Itaya et al 1982). Fabrication of electrochromic cells with single films of PB and mixed films of PB and other electrochromic materials has been demonstrated (Carpenter and Conell 1990; Leventis and Chung 1990; Mortimer 1991; Habib et al 1991). Electrochromism arises from the electronic transition at the redox centres of the PB film accompanied by the intercalanation/deintercalation of the hydrated alkali metal ions into/from the film. Ability of nonaqueous medium to influence the solvation sheath and hence the size of the cation has been successfully used in the development of fast switching electrochromic devices (Leventis and Chung 1991).

Another significant development in this functionalization of electrode surface leading to electrochromism is electrode modification with thin surface films of nickel hydroxide (Carpenter et al 1987; Govenstin et al 1990; Joseph et al 1991). A platinum surface is electrochemically modified with a NiHCF film and then dipped in $\mathrm{KOH}$ or $\mathrm{NaOH}$ to obtain a stable nickel hydroxide film. The modified electrode when cycled between 0 and $0.5 \mathrm{~V}$ (vs NCE) shows highly reversible redox behaviour ( $c f$ figure 6),

$$
\mathrm{Ni}(\mathrm{OH})_{2} \rightleftharpoons \mathrm{NiOOH}+\mathrm{H}^{+},
$$

and exhibits electrochromism with a sharp colour transition (black to colourless) which is highly reproducible and stable over prolonged cycling ( $3000 \mathrm{cycles})$. The switching time of the electrode is considerably low and is about $100 \mathrm{~ms}$ ( $c f$ figure 7).

Yet another functional characteristic exhibited by MHCF-modified electrodes is the photocurrent response, which may be useful in the design of photoelectrochemical devices. PB-modified electrode (Upadhyay et al 1991) is predominantly $n$-type and exhibits stable photocurrents whose polarity depends on the experimental conditions. 

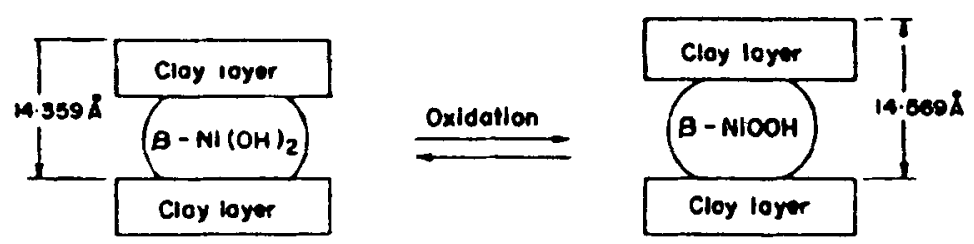

Figure 8. Scheme showing the reversible switching of the montmorillonite clay (host) due to the redox stimulus of the $\mathrm{Ni}(\mathrm{OH})_{2} / \mathrm{NiOOH}$ system.

Similarly, indium HCF modified electrode (Gomathi et al 1993) shows predominantly $p$ type behaviour and is characterized by enhanced photocurrents and inhibition of photocorrosion.

Recently, the possibility of designing an 'elastic' host which undergoes reversible volume changes in response to the changes in the guest species upon redox/electrochemical stimulus, has been demonstrated for the first time (Ravichandran et al 1996). The interlayer spacing of the host, montmorillonite clay, as revealed by X-ray diffraction data, undergoes reversible change (basal spacing $\left(d_{001}\right)$ changes by $0.237 \AA$ ) in response to the redox chemistry of $\mathrm{Ni}(\mathrm{OH})_{2} / \mathrm{NiOOH}$ system ( $c f$ figure 8 ). Such 'elastic' hosts, when coupled to electrodes can lead to novel applications of MLF on electrode surfaces.

\subsection{Conducting polymer modified electrodes}

Use of organic polymer coatings to introduce chemical functionality to the electrode surface is very well known. Multilayered, dynamic polymer coatings probably provide a three-dimensional reaction zone at the electrode surface. This gives rise to an increase in the flux of reactions that occur there, which in turn increases sensitivity. Moreover, polymer coatings with functional groups facilitate further chemical derivatization of the surface. Though the polymeric coatings on the electrode can be obtained by simple solution-casting, the method of electropolymerization which offers good control on rate and extent of polymerization is often the preferred method of synthesis. Polymeric modification, especially those of conducting polymers, have opened up new vistas in the functionalization of electrode surfaces. A few interesting results will be discussed below.

Polymers, particularly conducting polymers, are being widely used in the development of sensors. Their application in the biosensor development will be discussed later. An interesting development is the MLF of the polymer film on the electrode and thereby the introduction of selectivity to the modified electrode. Polypyrrole (PPy) film is electrochemically deposited on glassy carbon electrode and then subjected to electrochemical overoxidation (Freund et al 1991; Witkowski et al 1991). Overoxidation results in the modification of the polymer film surface by the introduction of carboxyl groups. These carboxyl functional groups impart charge-based selectivity to the modified electrode. This type of molecular level functionalization has enabled the discrimination of electroactive species when analysed voltammetrically. Thus, the overoxidized PPy film on GC electrode has been shown to suppress the $\mathrm{CV}$ response of ferro/ferricyanide and to distinguish between neurotransmitters, viz., ascorbic acid and dopamine. Similarly, polythiophene and poly(thiophene-3-acetic acid) films obtained on platinum by electrodeposition have been functionalized at the molecular level to exhibit charge selectivity (Chan et al 1995). The presence of electron-rich functionalities like carboxyl, 
carbonyl groups on the polymer surface is considered to be responsible for the chargeselectivity as in the case of oxidized PPy films.

Conducting polymer-modified electrodes, prepared from controlled electropolymerization conditions, are found to exhibit MLF characteristics. Thus, PPy-modified electrode incorporated with anionic complexing ligands, viz., alizarin red $S$, sulphasalicylic acid have offered scope for developing $\mathrm{Cu}(\mathrm{II})$ and $\mathrm{Cu}(\mathrm{I})$ selective electrodes (Shiu et al 1995).

\subsection{Crystalline conducting polymer}

Electrochemically synthesized polyparaphenylene (PPP) on indium-tin oxide glass has shown the promise of obtaining free-standing and crystalline films, which were not possible hitherto by chemical synthesis. This has been made feasible by microemulsionbased electrosynthesis which takes advantage of the templating property of the surfactant assemblies in directing the morphology of the PPP film (Tamilselvan et al 1995). X-ray diffraction studies of the PPP film at low thickness $\left(<2.35 \mathrm{mC} \mathrm{cm}^{-2}\right)$ reveal a highly reproducible crystalline structure of spherulite morphology. These highly 'molecularlyordered' spherulites are birefringent and hold the promise of producing polarized light and are used in electroluminescent devices.

\section{Enzyme modified electrodes}

Functionalization of electrodes with enzymes and biomolecules is an active area of current research. Enzymes, because of their very high specificity and sensitivity to substrates, facilitate the imparting of predesigned functional characteristics to the electrode surface. In this context, the tremendous progress in molecular recognition using enzyme-modified electrodes deserves special mention.

Following the success of coupling an enzyme-immobilized polymeric membrane to an oxygen electrode (Clark and Lyons 1962) for the detection of glucose, the technology of biosensors has grown rapidly in the last decade or so. The MLF of electrode surfaces with enzymes has been actively pursued. In the earlier version, electrodes were covered with an inert porous polymeric membrane into which enzyme was immobilized through chemical cross-linking. In the amperometric glucose sensor, constructed using the above approach, the electron transfer between the electrode and the FAD centre of the glucose oxidase (GOx) enzyme often proved to be sluggish because the FAD centre is invariably buried deep in the protein shell of the enzyme. Hence, to achieve facile electron transfer, redox mediators which act as efficient electron shuttles were introduced along with the enzyme into the membrane (Cass et al 1985). Attempts were made to functionalize the GOx enzyme, by derivatizing the enzyme with redox mediators like ferrocene and its derivatives (Degani and Heller 1987; Bartlett et al 1991). Ferrocene-modified PPy has also been employed to immobilize GOx for efficient electron transfer (Foulds and Lowe 1988). To facilitate rapid and direct electron transfer, molecular wiring of enzyme with the electrode is being actively pursued (Schuhmann et al 1991; Heller 1992; Ohara et al 1994).

Another significant development towards achieving direct electron transfer is the use of thiol SAM for molecular-level binding of the enzyme to the electrode. Glutathione reductase enzyme is covalently attached to the SAM of bis( $\mathrm{N}$-hydroxy succinimide ester) of 3,3'-dithiopropionic acid. This was subsequently modified with bipyridinium relay groups that facilitate direct electron transfer (Willner et al 1994). Moreover, this approach 
has also been shown to be successful for coupling photoexcitable species and thereby achieving the design of photocatalytic systems.

A remarkable development reported very recently (Riklin et al 1995) describes stripping the GOx of its FAD cofactor, modifying the FAD with ferrocene and reconstituting the apoprotein with modified cofactor. This approach is reported to make the electron transfer much more facile and reproducible than the earlier methods, which generally provide random contacts between the redox protein and the redox relay. Further, this method has been made more efficient by covalent linking of the reconstituted enzyme to the electrode through a thiol SAM. This SAM-based approach will have potential applications in the design of bioelectronic and optoelectronic devices.

\section{Conclusions}

The foregoing brief discussions reveal clearly the exciting developments in the field of MLF of electrode surfaces. It can be seen that MLF has (i) added new dimensions and versatility to electrochemistry, (ii) facilitated the predesigning of electrochemical interfaces to achieve sensitivity and selectivity in reactions, (iii) enhanced the applications of electrochemistry in catalysis and molecular recognition, and (iv) provided further insights into the mechanism of electron transfer. Further, the progress currently witnessed in the design of molecular electronic components like switches, gates, rectifiers, capacitors etc., seems to be encouraging indicators for the eventual realization of biocomputers in the 21 st Century. Finally, it is clear that only a beginning has been made; a great deal more needs to be unravelled.

\section{Acknowledgements}

The author expresses his sincere thanks to Dr G Prabhakara Rao for his valuable advice and suggestions and Drs S Bharathi, H Gomathi, J Joseph, K L N Phani, S Pitchumani, S Ravichandran, D N Upadhyay and other colleagues for their helpful discussions. He is grateful to Prof G V Subba Rao for his kind encouragement.

\section{References}

Bartlett P N, Bradford V Q and Whitaker R G 1991 Talanta 3857

Bharathi S, Yegnaraman V and Rao G P 1993 Langmuir 91616

Bharathi S, Yegnaraman V and Rao G P 1995 Langmuir 11666

Carpenter M K and Conell R S 1990 J. Electrochem. Soc. 1372464

Carpenter M K, Conell R S and Corrigan D A 1987 Solar Energy Mater. 16333

Cass, A E G, Davis G, Green M J and Hill H A O 1985 J. Electroanal. Chem. 190117

Chan H S O, Toh C S and Gan L M 1995 J. Mater. Sci. 5631

Cheng Q and Toth A B 1992 Anal. Chem. 641998

Clark L C and Lyons C 1962 Ann. N.Y. Acad. Sci. 10229

Colvin V L, Goldstein A N and Alivisatos A P 1992 J. Am. Chem. Soc. 1145221

Degani Y and Heller A 1987 J. Phys. Chem. 911285

Ellis D, Eckhoff M and Neff V D 1981 J. Phys. Chem. 851225

Finklea H O and Hanshew D D 1992 J. Am. Chem. Soc. 1143173

Foulds N C and Lowe C R 1988 Anal. Chem. 602473

Freund N, Bodalbhai L and Toth A B 1991 Talanta 3895

Gomathi H, Upadhyay D N and Rao G P 1993 Solar Energy Mater. 30161

Govenstin A, Decker F, Estrada W, Esteves C, Anderson A, Passerini S, Pantolani S and Scrosati B 1990 J. Electroanal. Chem. 277277 
Habib M A, Maheswari S P and Carpenter M K 1991 J. Appl. Electrochem. 21203

Heller A 1992 J. Phys. Chem. 963579

Hong F T 1989 Molecular electronics - biosensors and biocomputers (ed.) F T Hong (New York: Plenum) p. 105

Itaya K, Ataka T, Toshima S and Shinohara T 1982a J. Phys. Chem. 862415

Itaya K, Shibayama K, Akahoshi H and Toshima S 1982b J. Appl. Phys. 53804

Itaya K, Shoji N and Uchida I $1984 \mathrm{~J}$. Am. Chem. Soc. 1063423

Itaya K, Uchida I and Neff V D 1986 Acc. Chem. Res. 19162

Joseph J, Gomathi H and Rao G P 1991 Solar Energy Mater. 231

Joseph J, Gomathi H and Rao G P 1992 Bull. Electrochem. 886

Kaneko M, Hara S and Yamada Y 1985 J. Electroanal. Chem. 194165

Kellawi H and Rossiensky D R 1982 J. Electroanal. Chem. 131373

Kobayashi N, Hirohashi R, Ohno H and Tsuchida E 1990 Solid State Ionics 40/41 491

Krishnan V, Xidis A L and Neff V D 1991 Anal. Chim. Acta 2397

Kulesza P J and Doblhofer K 1989 J. Electroanal. Chem. 27495

Leventis N and Chung Y C 1990 J. Electrochem. Soc. 1373321

Leventis N and Chung Y C 1991 J. Electrochem. Soc. 138 L21

Malem F and Mandler D 1993 Anal. Chem. 6537

Miller C and Gratzel M 1991 J. Phys. Chem. 955225

Miller C, Cuendet P and Gratzel M 1991 J. Phys. Chem. 95877

Mortimer R J 1991 J. Electrochem. Soc. 138460

Mortimer R J and Rossiensky D R 1983 J. Electroanal. Chem. 151133

Neff V D $1978 J$. Electrochem. Soc. 125886

Ohara T J, Rajagopalan R and Heller A 1994 Anal. Chem. 662451

Porter M D, Bright T B, Allara D L and Chidsey C E D 1987 J. Am. Chem. Soc. 1093559

Ravichandran S, Phani K L N, Mani A and Pitchumani S 1996 J. Electroanal. Chem. 406227

Riklin A, Katz E, Willner I, Stocker A and Buckman A F 1995 Nature (London) 376672

Rubinstein I, Steinberg S, Tor Y, Shanzer A and Sagiv J 1988 Nature (London) 332427

Sagiv J 1980 J. Am. Chem. Soc. 10292

Schuhmann W, Ohara T J, Schmidt H L and Heller A 1991 J. Am. Chem. Soc. 1131394

Shiu K K, Chan O Y and Pang S K 1995 Anal. Chem. 672828

Takehara H and Takemura H 1995 Bull. Chem. Soc. Jpn. 681289

Tamilselvan S, Mani A, Pitchumani S and Phani K L N 1995 J. Electroanal. Chem. 384183

Troughton E B, Bain C D, Whitesides G M, Nuzzo R G, Allara D L and Porter M D 1988 Langmuir 4365

Upadhyay D N, Gomathi H and Rao G P 1991 J. Electroanal. Chem. 301199

Walczak M M, Chung C, Stole S M, Widrig C A and Porter M D 1991 J. Am. Chem. Soc. 1132370

Whitesides G M and Labinis P E 1990 Langmuir 687

Willner I, Lapidot N, Riklin A, Kasher R, Zahavy E and Katz E 1994 J. Am. Chem. Soc. 1161428

Witkowski A, Freund M S and Toth A B 1991 Anal. Chem. 63622 